\title{
Aspetti di criticità delle prove contenute nel volume "il test di italiano per stranieri"
}

Paolo Torresan

\section{Resumo:}

Através deste ensaio pretendemos fornecer as diretrizes para a construção de itens/evidência linguística. A operação foi desenvolvida ao negativo passando em revisão item e teste de um volume recém-publicado na Itália, voltado para aqueles que querem praticar testes semelhantes aos construídos para a liberação da autorização de residência. Há muitos aspectos que comprometem as provas contidas no volume em estudo:

- 2.1. Solução dupla

- 2.2. Solução de um item no item seguinte

- 2.3. Distractors que são mutuamente exclusivos

- 2.4. "Detachment" das teclas

- 2.5. Chaves de superposições

- 2.6. Ambiguidade dos distractors

- 2.7. Respostas óbvias

- 2.8. Contorções lógicas

- 2.9. Elicitação de julgamentos com base em habilidades não linguísticas

- 2.10. Opacidade do texto

- 2.11. Imagens difíceis de interpretar

- 2.12. Distúrbios de percepção

- 2.13. Incompatibilidade de imagens-texto

- 2.14. Busca de itens sujeitos a negação

- 2.15. Formulação deselegante do item

- 2.16. Não linearidade dos itens 
- 2.17. Sub-representação do construto

- 2.18. Opacidade dos exemplos e dos fornecimentos

- 2.19. Complexidade das entregas

- 2.20. Entregas de imprecisão

- 2.21. Confuso layout

- 2.22. Prompt de pouco generativa

- 2.23. Não calibrar os itens

- 2.24. Textos desactualizados a ser elaborado

- 2.25. Misprints

Palavras-chave: Italiano L2. Testing. Item Writing. Validade.

\begin{abstract}
:
Attraverso questo saggio intendiamo offrire delle linee-guida per la costruzione di item/prove linguistiche. L'operazione è svolta al negativo, passando in rassegna item e test di un volume recentemente pubblicato in Italia, rivolto a coloro che vogliono esercitarsi in prove simili a quelle costruite per il rilascio del permesso di soggiorno. Numerosi sono gli aspetti che inficiano la bontà delleprove contenute nel volume oggetto di studio:
\end{abstract}

- 2.1. Doppia soluzione

- 2.2. Soluzione di un item in item successivi

- 2.3. Distrattori che si escludono a vicenda

- 2.4. "distacco" delle chiavi

- 2.5. Sovrapposizione delle chiavi

- 2.6. Ambiguità dei distrattori

- 2.7. Ovvietà delle risposte

- 2.8. Contorsioni logiche

- 2.9. Elicitazione di giudizi basati su competenze non linguistiche

- 2.10. Opacità del testo 
- 2.11. Immagini difficili da interpretare

- 2.12. Fattori di disturbo percettivo

- 2.13. Mancata corrispondenza testo-immagini

- 2.14. Ricerca di elementi oggetto di negazione

- 2.15. Formulazione inelegante degli item

- 2.16. Mancata linearità degli item

- 2.17. Sottorappresentazione del costrutto

- 2.18. Opacità degli esempi e delle consegne

- 2.19 complessità delle consegne

- 2.20 imprecisione delle consegne

- 2.21. Layout confuso

- 2.22. Prompt poco generativi

- 2.23. Mancata calibrazione degli item

- 2.24. Inattualità dei testi da redigere

- 2.25. Refusi

Parole-chiave: Italiano L2. Testing. Item Writing. Validità.

\begin{abstract}
:
Through this essay we intend to provide the guidelines for the construction of items/linguistic evidence. The operation took place at the negative, reviewing item and test of a volume recently published in Italy, aimed at those who want to practice tests similar to those built for the release of the residence permit. There are many aspects that disrupt the goodness of the tests contained in the volume under study:
\end{abstract}

- 2.1. Dual solution

- 2.2. Solution of an item in the following item

- 2.3. Distractors that are mutually exclusive

- 2.4. "Detachment" of the keys 
- 2.5. Superimposition keys

- 2.6. Ambiguity of distractors

- 2.7. Obvious answers

- 2.8. Logical contortions

- 2.9. Elicitation of judgments based on non-linguistic skills

- 2.10. Opacity of the text

- 2.11. Images difficult to interpret

- 2.12. Perceptual disturbances

- 2.13. Mismatch text-images

- 2.14. Searching for items subject to denial

- 2.15. Inelegant formulation of item

- 2.16. Non-linearity of the items

- 2.17. Under-representation of the construct

- 2.18. Opacity of the examples and deliveries

- 2:19 complexity of deliveries

- 2.20. imprecision deliveries

- 2.21. Layout confusing

- 2.22. Prompt little generative

- 2.23. Failure to calibrate the items

- 2.24. Outdated texts to be drawn up

- 2.25. Misprints

Keywords: Italiano L2. Testing. Item Writing. Validity.

\section{Il test ufficiale di conoscenza della lingua italiana}

Il volume "Il test di italiano per stranieri" (di P. Avella, P. Borgonovo, F. Desiderio; 2013) rappresenta la seconda edizione dell'omonimo testo pubblicato nel 2011 a nome di Francesca Desiderio e Alberto Raminelli. 
Si presenta come uno strumento di sostegno per chi voglia superare il test ufficiale di conoscenza dell'italiano, previsto dall'art. 9 del decreto di legge n. 286 (25/7/1998) e regolato dal D.M. del 4.6.2010 (con integrazioni nelle circolari del Ministero dell'Interno n. 7589 del 16.11 .2010 e n. 8071 del 1.12.2010). I destinatari delle prove volute dal Ministero sono "cittadini non comunitari che, in possesso da almeno cinque anni di un permesso in corso di validità rilasciato in Italia e dei requisiti di reddito, intendono chiedere il rilascio del permesso di soggiorno CE per soggiornanti di lungo periodo" (AVELLA et al., 2013, p. xvi). Le prove del test ufficialesono calibrate sul livello A2 del Quadro di riferimento Europeo, valutano l'abilità di lettura, di ascolto e di scrittura (l'oralità è ignorata).

Il volume si presenta come uno strumento di esercitazione autonoma, al pari di altri testi relativi alle certificazioni linguistiche (nell'ambito dell'italiano LS, pensiamo ai volumi sul CILS o sul CELI editi da Guerra, o sul PLIDA editi da Alma Edizioni; nell'ambito dell'inglese LS si pensino alle simulazioni delle prove del TOEFL o dell'IELTS). C’è però una differenza sostanziale: gran parte delle prove presenti nel volume oggetto di studiosono pensate a tavolino dalle autrici, non sperimentate con candidati del test ufficiale di italiano. Fanno eccezione due sole prove (una somministrata a Firenze il 17/01/2011 e l'altra ad Aosta il 25/10/2012), pubblicizzate nella copertina come "vere" (i.e. realmente somministrate).

\section{L'analisi}

La presenza di item inadeguati presenti nel volume oggetto di analisi consente di mettere in evidenza una serie di passi falsi che un valutatore dovrebbe evitare. Consideriamo nei paragrafi che seguono ciascun elemento critico, commentandolo e talora suggerendo possibilità di emendamento.

\subsection{Doppia soluzione}

Si consideri il seguente esercizio (p. 51):

RIORDINA LE SEGUENTI FRASI E RICOMPONI IL BRANO 
Aspetti di criticità delle prove contenute nel volume "il test di italiano per stranieri" Paolo Torresan

a. Solo di pomeriggio tardi la situazione è migliorata.

b. Ieri mattina, come in molte città italiane, è arrivata la prima nevicata anche a Torino.

c. Come sempre ci sono stati molti problemi:

d. Per questo molte persone si sono arrabbiate con il Comune.

e. autobus e tram hanno avuto grandissimi ritardi e si sono formate lunghe file di macchine.

La soluzione prevista dalle autrici è:

- Ieri mattina, come in molte città italiane, è arrivata la prima nevicata anche a Torino.

- Come sempre ci sono stati molti problemi:

- autobus e tram hanno avuto grandissimi ritardi e si sono formate lunghe file di macchine.

- Per questo molte persone si sono arrabbiate con il Comune.

- Solo di pomeriggio tardi la situarione è migliorata.

Secondo noi, tuttavia, pure questa combinazione è ammessa:

a. Ieri mattina, come in molte città italiane, è arrivata la prima nevicata anche a Torino.

b. Solo di pomeriggio tardi la situazione è migliorata.

c. Come sempre ci sono stati molti problemi:

d. autobus e tram hanno avuto grandissimi ritardi e si sono formate lunghe file di macchine.

e. Per questo molte persone si sono arrabbiate con il Comune.

Un problema analogo si riscontra nella prova che segue (p. 57): lo studente deve leggere il testo e individuare l'informazione appropriata (è una sorta di vero/falso). Coloro che hanno confezionato la prova ritengono che la soluzione sia costituita da un solo item, il\# 2. A nostro avviso sono ammissibili, invece, entrambe le alternative. Siamo convinti che l'annuncio di cui si parla riguardi sì in generale tutte le persone che passeranno l'estate in città (\#2), tuttavia viene fornita un'informazione utile anche a chi ama la danza (\# 1).

Il presunto distrattore costituisce una 'verità parziale', di conseguenza può originare confusione.

Sono molte le iniziative e le attività che il Comune ha organizzato per chi passa l'estate in città. Mostre, concerti di musica classica e jazz all'aperto, danza 
classica e moderna. Inoltre, molti musei resteranno aperti fino alle 22 e, dal 12 gingno al 30 settembre, con un solo biglietto sarà possibile visitarne due a scelta.

Questa informazione è rivolta a:

1. Le persone che amano la danza

2. Le persone che trascorreranno l'estate in città.

Il problema si ripete nella provaqui sotto (p. 56): perfino un madrelingua può rimanere perplesso su quale alternativa scegliere.

Esce in edicola una raccolta di canzonidei più importanti cantautori degli anni 70. A partire dal $1^{\circ}$ settembre, per 12 settimane, ogni settimana si potrà acquistare un $C D$ doppio a soli 9,90 euro. Ogni confezione contiene anche un fascicolo con tante immagini, le biografie degli autori e $i$ testi delle canzoni. Un'occasione da non perdere!

La raccolta contiene:

1. I grandi successi musicali degli anni '70

2. Canzoni, testi e immagini di importanti musicisti italiani

Carr scriveal riguardo (2011: 91): "There should be one option that is clearly correct, not two or more that are partially correct".

\subsection{Soluzione di un item in item successivi}

Se si scorrono gli item a scelta multipla che seguono (p. 75), facenti capo ad uno stesso esercizio, ci si accorge che è possibile rispondere all'item \#1, considerando l'informazione presente nello stem dell'item \#3:

1. Gli antibiotici:
a. Si possono acquistare solo con la ricetta del medico
b. Si possono acquistare liberamente in farmacia
c.Si possono acquistare liberamente al supermercato

2. In caso di influenæa: 

a. Si possono chiedere antibiotici direttamente al farmacista
b. Gli antibiotici non sono necessari
c.Bisognastare a letto finché non è passata

\section{Se il medico prescrive un antibiotico:}

a. Si può utilizzare quello che abbiamo in casa, perché uno vale l'altro

b. Ė meglio ignorare la prescrizione

c.Si deve assumere seguendo esattamente tempi e modi indicati dal medico

Non è dunque necessario leggere la porzione di testo cui l'item \#1 si riferisce per selezionare l'opzione corretta.

Nel caso qui sotto, invece (p. 60-61), si può dedurre la soluzione dell'item \#1 attraverso la lettura degli item successivi: da quelli si capisce che sono molti (e non pochi, come recita il distrattore dell'item \#1) i ricordi legati all'infanzia dell'intervistato. Quindi, se lo studente, ad una prima lettura del testo di riferimento, avesse risposto in maniera non corretta allitem \#1, potrebbe esser in grado di modificare la propria scelta attraverso una deduzione a partire dalla lettura degli item successivi.

1. L'intervistato:

a. Ha pocbi ricordi della sua infanzia

\section{b. Ricorda con piacere la sua infanzia}

2. In estate l'intervistato:
a. Restava spesso in città
b. Passava qualche mese al mare

3. L'intervistato:
a. Non amava la scuola
b. Amava leggere e disegnare

4. L'intervistato, da bambino: 

a. Guardava molti film in bianco e nero
b. Preferiva fare sport con gli amici

5. Quando era bambino l'intervistato:
a. Amava andare in bicicletta
b. Non amava il Natale

\section{L'intervistato:}
a. Ha avuto un'infanzia felice
b. Non si divertiva

In quest'ennesimo caso(p. 81) l'ultimo item (\# 7) ci informa che l'item precedente (\# 6) è vero.

1. Akiko non ha il permesso di soggiorno

$\begin{array}{ll}V & F \\ V & F \\ V & F \\ V & F \\ V & F \\ V & F \\ V & F\end{array}$

Un item writer, tuttavia, dovrebbe essere tenutoa garantire l'indipendenza di un item rispetto agli altri (Item Independence, cfr. Carr 2011).

\subsection{Distrattori che si escludono a vicenda}

Nell'item che segue (p. 52), anche senza leggere il testo di riferimento, è facile stabilire che la risposta è a), dal momento che i distrattorisi autoescludono (due sorelle sono, per forza di cose, due persone diverse!).

1. Lella e Gabriella:
a. Sono la stessa persona
b. Sono due persone diverse
c.Sono due sorelle 


\section{4. "Distacco" delle chiavi}

Le chiavi si distaccano rispetto ai distrattori quando vengono formulate con frasi più ampie e complesse (Heaton 1988). Si vedano gli esempi, evidenziati in grassetto.

\section{LEGGI IL BRANO E RISPONDI ALLE DOMANDE}

\section{SEGUENTI}

Il vaccino influenzale

Ogni anno, in inverno, la popolarione italiana è colpita da una epidemia di influenza. Si tratta di una malattia assolutamente non grave, che può essere pericolosa solo per alcune categorie di persone. In questi casi, la maniera migliore per prevenire e combattere l'influenza è il vaccino.

Dato che l'influenza aumenta la probabilità di contrarre altre inferioni, questa malattia può essere pericolosa per gli anziani e per chi soffre di malattie croniche. Il ministero della Salute raccomanda quindi la vaccinazione a coloro che hanno più di 64 anni, a chi è in frequente contatto con gli anziani, a ci soffre di malattie respiratorie e polmonari, di diabete, di disfunzioni renali e di altre malattie croniche.

\section{L'influenza}

a. È una malattia molto grave

\section{b. Può essere pericolosa solo per persone anziane e debilitate}

c. Ė una malattia ormai debellata

d. Può essere curata con il vaccino

2. A chi è raccomandato il vaccino influenzale?

a. Tutti dovrebbero fare la vaccinazione!

b. Nessuno si dourebbe vaccinare

c. Solo ai bambini molto piccoli 


\section{d. Agli anziani e a chi soffre di malattie respiratorie croniche}

Alderson et al. (1995: 49) ammoniscono: "The correct alternative should not look so different from the distractors that it stands from the rest. It should not be noticeably longer or shorter, nor be written in a different style".

\subsection{Sovrapposizione delle chiavi}

Nel caso di cui sopra, inoltre, le due chiavi hanno di per sé un potere attrattivo in quanto si sovrappongono, rimandandoa una stessa informazione: nel caso dell'influenza, gli anziani sono categorie a rischio.

Carr scrive (2011: 89) a riguardo:"Two items should not pose the same question, even with slightly different wording".

Nelle Guidelines for Writers of Reading Test, allestite dal British Council ungherese, all'interno del Progetto Into Europe (2002), a cui si può accedere mediante il sito dell'Università di Lancaster (cfr. sitografia), il concetto è espresso sinteticamente: "Two items must not have similar answers" (si veda anche Cangelosi 1990).

\subsection{Ambiguità dei distrattori}

Tornando a considerare l'esercizio al $\$ 2.4$., notiamo che il distrattore d) del primo item(vedi sotto) può confondere chi si accinge a svolgere la prova Immaginiamo che unlettore non sappia che cosa sia il "vaccino". Dal testo coglie che il vaccino serve a "prevenire e combattere l'influenza". Il distrattore d) lo avvisa che l'influenza "può essere curata con il vaccino". Ipotizzando, appunto, che la "prevenzione" sia un concetto assodato, può far corrispondere il concetto di "combattere" a quello di "curare".

Il vaccino influenzale

Ogni anno, in inverno, la popolarione italiana è colpita da una epidemia di influenza. Si tratta di una malattia assolutamente non grave, che può essere 
Aspetti di criticità delle prove contenute nel volume "il test di italiano per stranieri" Paolo Torresan

pericolosa solo per alcune categorie di persone. In questi casi, la maniera migliore per prevenire e combattere l'influenza è il vaccino.

Dato che l'influenza aumenta la probabilità di contrarre altre inferioni, questa malattia può essere pericolosa per gli anziani e per chi soffre di malattie croniche. Il ministero della Salute raccomanda quindi la vaccinazione a coloro che banno più di 64 anni, a chi è in frequente contatto con gli anziani, a chi soffre di malattie respiratorie e polmonari, di diabete, di disfunzioni renali e di altre malattie croniche.

1. L'influenza

a. È una malattia molto grave

b. Pù essere pericolosa solo per persone anziane e debilitate

c. È una malattia ormai debellata

d. Può essere curata con il vaccino

\subsection{Contorsioni logiche}

Haladyna et. al 2002 consigliano di evitare l'uso di negazioni nella formulazione degliitem, dal momento che rendono difficile la comprensione. Nell'esercizio di vero/falso che segue (pp. 80-81) lo studente deve compiere una piroetta logica, dal momento che la risposta corretta prevede di ragionare sulla negazione di una negazione.

Impiegata: Buongiorno, come posso aiutarla?

Akiko: $\quad$ Ho letto il vostro annuncio sul giornale e sono venuta per vedere se ci sono opportunità di lavoro per me.

Impiegata: Bene, Lei ha un permesso di soggiorno?

Akiko: $\quad$ Si, di studio, per tre anni, frequento una scuola di italiano.

1. Akiko non ba il permesso di soggiorno $V \quad \boldsymbol{F}$

\subsection{Risposte ovvie}


$\mathrm{Nel}$ seguente test ad individuazione di informazioni lo studente deve evidenziare quali sono le affermazioni "corrette", i.e. corrispondenti a contenuti presenti nel testo (p. 61). Rileviamo la presenza di un item ovvio, l'item \#1, cui si può rispondere sulla base della propria conoscenza del mondo, a prescindere dal testo cui è riferito.

Insonnia, amore mio

Per molti italiani, la notte è un serio problema. Un problema che si chiama insonnia e che non permette di dormire bene, o semplicemente di dormire, a circa 12 milioni di italiani su 60. Troppo lavoro, fumo, alcol, stress, ma anche problemi fisici congeniti possono essere all'origine di questo disturbo. Molte persone afflitte da questo problema hanno difficoltà a concentrarsi durante lo studio o il lavoro. Ma non solo. Sono più nervose, aggressive e hanno difficoltà a prendere decisioni. Non dimentichiamo, inoltre, che dormire poco o male è all'origine di parecchi incidenti automobilistici.

\section{Chi soffre d'insonnia non dorme bene}

2 La maggioranza degli italiani pensa di aver problemi a dormire

3 Un italiano su 5 ba problemi di insonnia

4 Bere troppo può causare l'insonnia

5 Generalmente, chi soffre d'insonnia, durante la giornata è molto calmo

6 Chi studia ba meno problemi d'insonnia

7 Gli automobilisti, in generale, non corrono rischi perché possono dormire in macchina

È ovvia anche la risoluzione di questo item (p. 74):

1. I sacchetti di plastica:

a. sono comodi e non inquinano

b. sono comodi ma vanno ad aumentare la massa di rifiuti da smaltire

Lo stesso dicasi per il seguente (p. 116):

1. Al mercatino dell'usato si può trovare:

\section{a. abbigliamento}


Aspetti di criticità delle prove contenute nel volume "il test di italiano per stranieri" Paolo Torresan

b. cibo

Occorre fare in modo che gli item costringano il lettore a ritornare sul testo e a esso si riferiscano per poter rispondere (Passage Dependence; cfr. Keenan et al. 2008). Alderson et al. (1995: 50) dichiarano: "Some items do not test what they are intended to test. This most frequently occurs in comprehension tests, where items may turn out to be testing background knowledge. It is unfortunately easy to write item which can be answered without any reference to the reading or listening passage".

\subsection{Elicitazione di giudizi basati su competenze non linguistiche}

Nel test relativo all'insonnia di cui abbiamo discusso poc'anzi si riscontra la presenza di un item la cui risoluzione dipende da ragionamenti di tipo matematico: è l'item \#3.

Insonnia, amore mio

Per molti italiani, la notte è un serio problema. Un problema che si chiama insonnia $e$ che non permette di dormire bene, 0 semplicemente di dormire, a circa 12 milioni di italiani su 60. Troppo lavoro, fumo, alcol, stress, ma anche problemi fisici congeniti possono essere all'origine di questo disturbo. Molte persone afflitte da questo problema banno difficoltà a concentrarsi durante lo studio o il lavoro. Ma non solo. Sono più nervose, aggressive e banno difficoltà a prendere decisioni. Non dimentichiamo, inoltre, che dormire poco o male è all'origine di parecchi incidenti automobilistici.

1 Chi soffre d'insonnia non dorme bene

2 La maggioranza degli italiani pensa di aver problemi a dormire

\section{Un italiano su 5 ha problemi di insonnia}

4 Bere troppo può causare l'insonnia

5 Generalmente, chi soffre d'insonnia, durante la giornata è molto calmo

6 Chi studia ba meno problemi d'insonnia

7 Gli automobilisti, in generale, non corrono rischi perché possono dormire in macchina 
In tal caso uno studente, pur conoscendo bene la lingua oggetto di studio, potrebbe rispondere in maniera sbagliata per via di difficoltà di calcolo $(12 \times 5=60)$. Abbiamo, così, una situazione di Construct Irrelevant Variance: un elemento esterno a ciò che ci si propone di misurare può alterare la performance dello studente, e quindi compromettere la validità della prova.

\subsection{Opacità del testo}

Leggiamo il testo che segue (p. 79-80):

Centro di mediazione linguistica e culturale di Torino ricerca 1 MADRELINGUA EGIZIANO e 1 MADRELINGUA CINESE per attività di ufficio e consulenza. Requisiti: età da 20 a max. 40 anni; esperienza nel settore: [refuso $\rightarrow$;] diploma di scuola media e/ o laurea; conoscenza della lingua italiana; capacita di lavorare in gruppo e a contatto con le persone; capacità di ascoltare e capire i problemi delle persone. Inviare il CV via mail allindirizzoselezione@cmlc.it

\section{Puoi rispondere all'annuncio se:}

\section{1. sei di nazionalità marocchina? sì no \\ 2. preferisci stare solo? \\ si no}

A prescindere da un problema di layout (la prova è un vero/falso, quindi le frasi dovrebbero essere scritte per esteso, come in genere avviene, e non con uno stem e due completamenti come se fosse un esercizio di discriminazione) riscontriamo un problema nel momento in cui ci si trova a rispondere allitem evidenziato.

A chi si sottopone alla prova viene chiesto se uno studente marocchino può rispondere all'annuncio. Nell'annuncio si rende noto che "il [un?] centro di mediazione linguistica e culturale di Torino ricerca 1 madrelingua egiziano". Qui sorgono i problemi: cosa significa "madrelingua egiziano"? La nostra conoscenza del mondo ci fa dire che esistono cittadini egiziani che parlano una variante dialettale dell'arabo. Ora, se gli autori del testo avessero voluto dire che il centro di mediazione aveva bisogno di un nativo egiziano in grado di mettersi in relazione 
con i compatrioti, avrebbero dovuto spiegarsi meglio: "madrelingua arabo di nazionalità egiziana", dal momento che -ripetiamo- un egiziano e un marocchino, come un libanese e un giordano, o un saudita e uno yemenita- sono madrelingua arabi!

Poiché il testo non è trasparente, al momento di rispondere all'item \#1, chi esegue la prova può essere confuso.

\subsection{Immagini difficili da interpretare}

È invalsa l'opinione, tra gli insegnanti di lingua, che l'uso di immagini sia appropriato in un contesto valutativo. Si pensa, cioè, che le immagini costituiscano un linguaggio universale.

In realtà un'immagine è sensibile a interpretazioni diverse, per via di fattori individuali e culturali. Peggio ancora se è oscura, poco nitida o di cattiva qualità: non aggiunge alcun contributo alla prova, piuttosto è il contrario.

Nel seguente compito di scrittura (p. 100) lo studente è invitato a descrivere alcuni piatti sulla base di immagini poco chiare (anche uno studente di livello avanzato, e forse addirittura un italiano madrelingua, avrebbero difficoltà a capire a cosa il secondo e il terzo piatto si possano riferire).

OSSERVA LE IMMAGINI E DESCRIVI I DIVERSI PLATTI RAPPRESENTATI

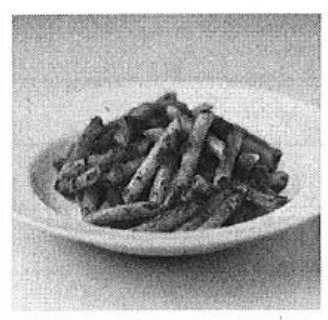

A

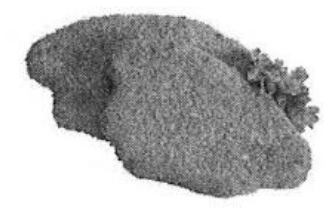

B

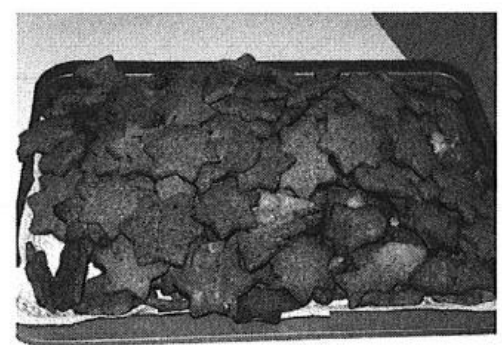

C

La figura $A$ rappresenta ..., mentre la $B \grave{e}$..., e laC ... 


\subsection{Fattori di disturbo percettivo}

Nella prova qui sotto l'allievo è tenuto a scrivere quali attività svolge, di solito, nei tre momenti della giornata (p. 104). Le doppie linee delle lancette e le cerchiature dei numeri costituiscono, a nostro avviso, fattori di disturbo percettivo.

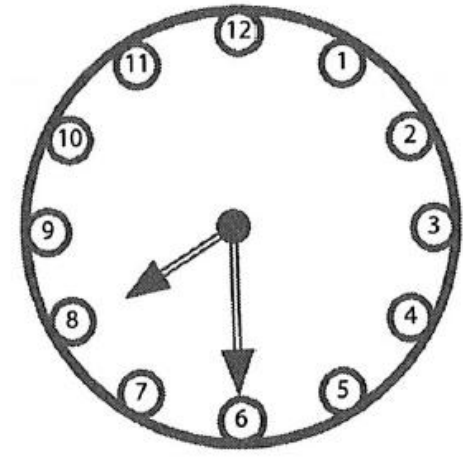

Mattina

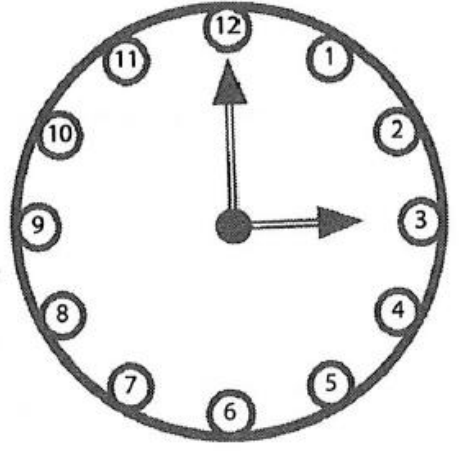

Pomeriggio

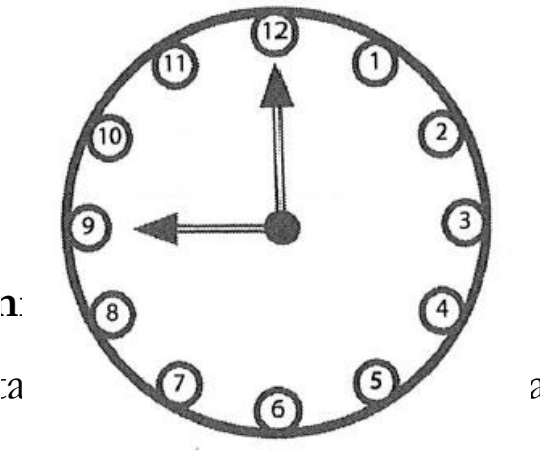

Sera

a

(mentre nel testo si fa riferimento alsenso di marciainverso; oltretutto, si badi, il divieto di sorpassare pare contravvenuto!);

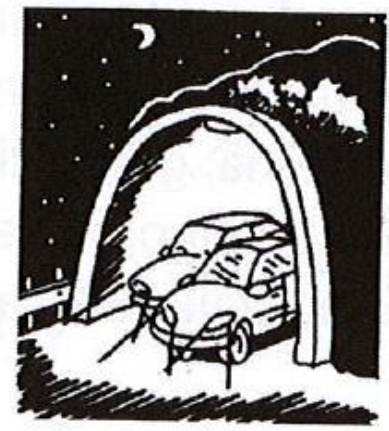

Di notte, quando si guida passando dal buio all'illuminazione delle gallerie, diminuisce la visibilità. $\grave{E}$ quindi consigliabile, prima di entrare nel tunnel, di rallentare e non sorpassare.

nel secondo caso, non c'è traccia di "alternanza nuvole e sole" al nord nell'immagine corrispondente.

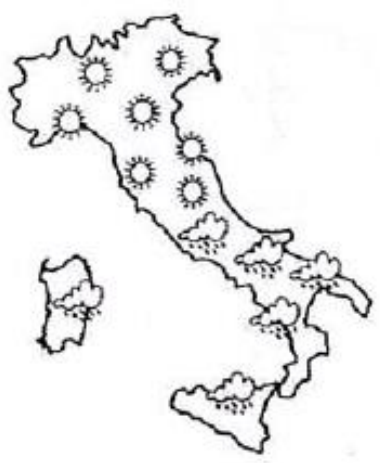

Previsioni metereologiche per il prossimo fine settimana: ci sarà un'alternanza di nuvole e sole al nord, invece, nel resto d'Italia continuerà la pioggia. 


\subsection{Ricerca di elementi negativi}

Nell'attività da cui sono stati tratti gli item qui sotto lo studente deve abbinare frasi a immagini.

L'anomalia di questi item (a cui, per comodità abbiamo già fatto corrispondere le immagini riferite) risiede nel fatto che il matching si basi su termini soggetti a una negazione.

Siamo convinti che ciògeneri confusione nella testa deltest taker (e forse anche in quella di un nativo), il quale ragiona in termini semantici e non lessicali, e quindi è portato a cercare nei disegni quanto affermato dalle frasi, e non quanto negato.

\section{COLLEGA LE SEGUENTI FRASI CON LE IMMAGINI}

\section{CORRISPONDENTI}

I miei figli non guardano tanta $t v$, preferiscono giocare in cortile

Ormai, per scrivere, non si usa più la penna, ma solo il computer...

\subsection{Formulazione inelegante degli item}

Si considerino i seguenti item di un test di lettura a scelta multipla (p. 75):

\section{Gli antibiotici:}
a. si possono acquistare solo con la ricetta del medico
b. si possono acquistare liberamente in farmacia
c. si possono acquistare liberamente al supermercato

A nostro avviso sarebbe più elegante la formulazione che segue:

1 Gli antibiotici si possono acquistare

a. solo con la ricetta del medico

b. liberamente in farmacia 


\section{C. liberamente al supermercato}

Allo stesso modo, nell'item che segue (p. 54),

1 Domenica 6 marzo:

a. sarà possibile cucinare cibo indiano

b. sarà possibile ascoltare musica indiana

C. sarà possibile assaggiare cibo indiano

potremmo intervenire con una resa più sintetica ed efficace:

1. Domenica 6 marzo sarà possibile:
a. cucinare cibo indiano
b. ascoltare musica indiana
c. assaggiare cibo indiano

\subsection{Mancata linearità degli item}

Nell'esercizio qui sotto si nota una mancata linearità degli item: non seguono lo sviluppo del testo. L'ultimo item costringe, infatti, a un testa-coda nella lettura: l'occhio deve risalire e scorrere le prime righe in cerca della risposta. Questo zigzagare dell'attenzione può generare confusione, perché viola una prassi cui il lettore è abituato: corrispondenza lineare item-testo (la situazione qui, oltretutto, è aggravata da una certa cripticità dello stesso item \#3).

La prima colarione

Tutti gli esperti di alimentazione sono d'accordo sul fatto che la colazione, il primo pasto della giornata, debba essere ricco e bilanciato. Una prima colazione completa dovrebbe comprendere, oltre al caffe e al tè che tutti beviamo, latte, yogurt, succhi di frutta, pane o altri derivati dei cereali (per esempio fiocchi di granoturco), burro in piccole quantità, marmellate, miele, formaggi freschi. Questi sono tutti alimenti adatti alla prima colarione, ma naturalmente non è opportuno mangiare tutte queste cose in un unico pasto! Una prima colazione energetica è necessaria per iniziare in modo corretto la giornata, ma se si hanno problemi di sovrappeso bisogna scegliere alimenti meno ricchi di calorie, come latte e yogurt e scremati, evitare il burro e consumare pane magro e pochi zuccheri. 
1. La prima colazione dourebbe comprendere sempre:

a. alimenti grassi come pancetta e salumi

b. alimenti ricchi di carboidrati, come pane, biscotti o fette biscottate

2. Chi ha problemi di sourappeso:

a. dovrebbe scegliere alimenti meno ricchi di calorie

b. dourebbe saltare la colazione

\section{Fare una ricca prima colazione:}

a. è importante, perché quello che si mangia al mattino non fa ingrassare

\section{b. è importante, perché è un pasto a tutti gli effetti}

\subsection{Sottorappresentazione del costrutto}

In merito alla competenza di "interazione scritta in generale" prevista per un livello A2, le indicazioni del Quadro di Riferimento Europeo (Council of Europe, 2001) sono le seguenti: "È in grado di scrivere brevi e semplici appunti relativi a bisogni immediati, usando formule convenzionali".

Nell'esercizio che segue (p. 98) gli appunti, in realtà, sono già dati; l'apprendentedeve solo agire a livello morfologico.Non è prevista quindi alcuna attività ideativa, di pianificazione, quindi di organizzazione semantica. Si tratta, insomma, di un esercizio di grammaticalizzazione di appunti; vale più a misurare la competenza morfolessicale che non l'abilità di scrittura.

Ciò mette in discussione la validità della prova: il costrutto (vale a dire l'oggetto della nostra stima: lo scrivere, in questo caso) è sottorappresentato (Construct Underrepresentation).

A ciò si aggiunga, per inciso, che vi è uno slittamento temporale: nelle consegne ci si riferisce allo ieri, mentre nel prompt (vale a dire nella traccia, nell' incipit volto a facilitare la stesura del testo) il punto di riferimento è l'oggi. 
Ieri hai avuto una giornata molto intensa; racconta a un amico cosa bai fatto, seguendo le informazioni scritte sul biglietto. Scrivi almeno 50 parole.

8.30 Accompagnare Anna Asilo

9 Pane e verdure

11 Macelleria

12 Preparare pran₹o

16 Telefonare a Luisa

19 Pizza

Ciao Filippo,

oggi ho avuto molti impegni:

\subsection{Opacità degli esempi e delle consegne}

Nell'attività di produzione scritta che segue (p. 100) la consegna è poco chiara: al test taker può non risultare evidente che è tenuto a scrivere dei messaggi diversi da quello fornito nell'esempio.

Del resto la presenza di attività di grammaticalizzazione nel set di task per la scrittura (come visto poc'anzi) può confondere il test taker, facendogli sorgere il dubbio: "Devo per caso intervenire sulla forma delle frasi presentate come esempio? Le devo riscrivere? Devo scriverne di altre?"'

Ci sovviene una annotazione di Alderson et al.(1995: 47): "Often students fail a test or an item not because their language is poor, but because they do not understand what they are meant to $d o "$.

STATE PARTENDO PER LE VACANZE E DOVETE

LASCIARE UN MESSAGGIO AI VOSTRI FIGLI, COME

NELL'ESEMPIO. CHE COSA SCRIVETE? 


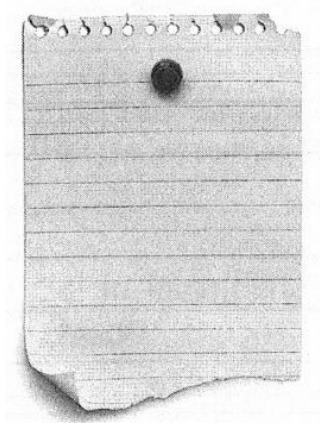

Ricordatevi di:

- Innaffiare le piante ogni due giorni

- Lavare gli asciugamani

- Fare la spesa: caffè, dentifricio, pasta e riso...

Ricordatevi di:

\subsection{Complessità delle consegne}

Riteniamo che il lessico con cui le consegne sono formulate nell'esercizio che segue (p. 89) sia complesso, vale a dire sovradimensionato rispetto alle competenze di uno studente A2 (vedi termini evidenziati in grassetto):

Stai cercando un aiutante per la tua attività, scrivi un annuncio. Scegli tra $i$ seguenti lavori: commesso/a, segretaria, magazziniere.

Descrivi:

- il settore e il tipo di lavoro

- i compiti e le mansioni da svolgere;

- i requisiti necessari;

- il tipo di contratto offerto

- i giorni e gli orari di lavoro

Scrivi almeno 50 parole.

\subsection{Imprecisione delle consegne}

Nel seguente esercizio (p. 75) la consegna è fuorviante. Gli studenti sono invitati a "rispondere alle domande"; in realtà, devono scegliere l'opzione corretta: 
il completamento della frase, nel primo caso; la risposta alla domanda, nel secondo caso.

\section{LEGGI IL BRANO E RISPONDI ALLE DOMANDE}

\section{SEGUENTI}

Il vaccino influenzale

Ogni anno, in inverno, la popolazione italiana è colpita da una epidemia di influenza. Si tratta di una malattia assolutamente non grave, che può essere pericolosa solo per alcune categorie di persone. In questi casi, la maniera migliore per prevenire e combattere l'influenza è il vaccino.

Dato che l'influenza aumenta la probabilità di contrarre altre inferioni, questa malattia può essere pericolosa per gli anziani e per chi soffre di malattie croniche. Il ministero della Salute raccomanda quindi la vaccinazione a coloro che banno più di 64 anni, a chi è in frequente contatto con gli anziani, a chi soffre di malattie respiratorie e polmonari, di diabete, di disfunzioni renali e di altre malattie croniche.

\section{L'influenza}
a. Ė una malattia molto grave
b. Pù̀ essere pericolosa solo per persone an₹iane e debilitate
c. È una malattia ormai debellata
d. Pù essere curata con il vaccino

2. A chi è raccomandato il vaccino influenzale?
a. Tutti dourebbero fare la vaccinazione!
b. Nessuno si dourebbe vaccinare
c. Solo ai bambini molto piccoli
d. Agli anziani e a chi soffre di malattie respiratorie croniche

Il problema si ripete in un esercizio cui abbiamo dato menzione (p. 80-81). Si tratta di un dialogo a cui seguono delle frasi; lo studente deve decidere quali sono vere e quali false. La consegna più appropriata sarebbe quella che invita 
l'esaminando a indicare se le frasi corrispondono a verità o meno; non deve rispondere ad alcun quesito, come invece si legge nelle istruzioni qui sotto.

AKIKO STA CERCANDO LAVORO ED E ANDATA IN UN'AGENZIA SPECIALIZZATA. LEGGI IL DIALOGO TRA AKIKO

E L'IMPIEGATA E RISPONDI ALLE DOMANDE.

Impiegata: Buongiorno, come posso aiutarla?

Akiko: $\quad$ Ho letto il vostro annuncio sul giornale e sono venuta per vedere se ci sono opportunità di lavoro per me.

Impiegata: Bene, Lei ba un permesso di soggiorno?

Akiko: $\quad$ Sì, di studio, per tre anni, frequento una scuola di italiano. $[\ldots]$

1. Akiko non ha il permesso di soggiorno

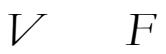

2. Akiko ba visto su internet un annuncio dell'agenzia

3. In passato, Akiko ba lavorato come cameriera

4. L'impiegata richiede un curriculum ad Akiko

5. Akiko ha studiato italiano

6. L'impiegata fissa un appuntamento con Akiko

V F

7. L'incontro è fissato per le 15

\subsection{Layout confuso}

Nella prova di lettura che segue (p.77) siamo convinti che la grafica, con l'alternanza di righe bianche e di righe con fondino, possa generare confusione nel test taker. È possibile l'allievo completi solo alcune di queste righee non le altre. In effetti, anche se nella consegna si parla di "tabella", il format di cui ci si avvale non è certo tra $i$ più diffusi.

LEGGI LA BIOGRAFIA DI RITA LEVI-MONTALCINI E

RIEMPI LA TABELLA CON LE INFORMAZIONI CHE TROVI DI SEGUITO

Rita Levi-Montalcini (Torino, 22 aprile 1909-Roma, 30 dicembre 2012), premio Nobel per la medicina nel 1986, è stata una delle più celebri ricercatrici 
Aspetti di criticità delle prove contenute nel volume "il test di italiano per stranieri" Paolo Torresan

italiane. Nel 1936 si laurea a Torino con il massimo dei voti; durante il fascismo vive per un periodo a Bruxelles e poi a Firenze. Al termine della guerra parte per St. Luis, negli Stati Uniti, dove diviene professore ordinario. Per circa trent'anni svolge questa professione, fin al premio Nobel per le sue ricerche sul sistema nervoso periferico. Nel 2001 viene nominata senatrice a vita.

Nome

Cognome

Anno di nascita

Luogo di nascita

Professione

Ha lavorato a

Riconoscimenti

Cariche politiche

\subsection{Prompt poco generativi}

I prompt (i.e.: stimoli per la produzione) che seguono (p. 93-94) paiono poco motivanti, perché:

- poco autentici (è improbabile che l'atto di comparare oggetti rientri tra i bisogni comunicativi impellenti di un apprendente di livello A2)

- richiedono una competenza lessicale elevata

OSSERVA LE IMMAGINI E DESCRIVI I DUE TAVOLI: QUALI DIFFERENZE CI SONO TRA DI ESSI? USA AL MASSIMO 30 PAROLE.

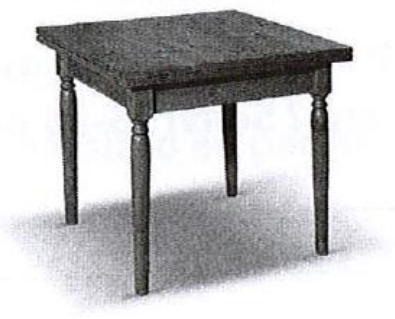

A

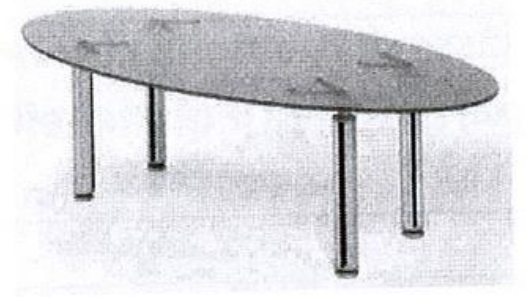

B 


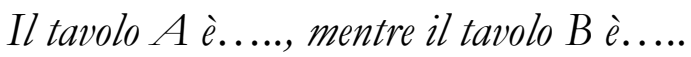

OSSERVA LE IMMAGINI E DESCRIVI LE DIFFERENZE TRA LE BICICLETTE RAPPRESENTATE. USA AL MASSIMO 30 PAROLE.

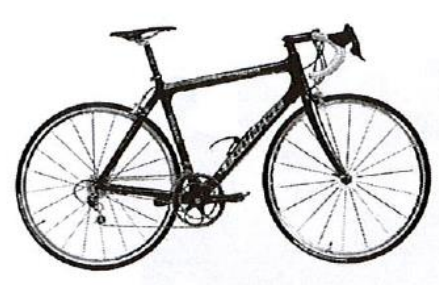

A

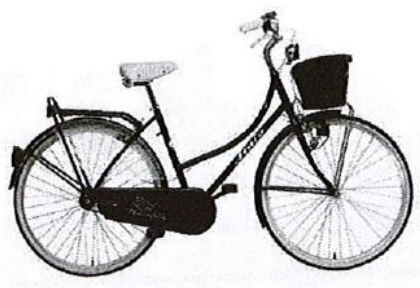

B

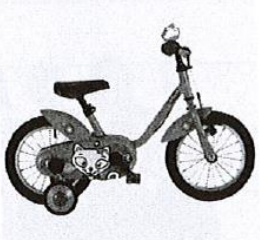

C

La bici $A$ è....., mentre la bici $B$ è....., e quella $C \grave{e} . .$.

A confermare l'inadeguatezza del compito sono, peraltro, i testi presentati dalle autrici come modello nella sezione "soluzioni"; in grassetto evidenziamo il lessico che uno studente di livello A2 difficilmente è in grado di usare:

Il tavolo A è quadrato e antico; basso e di legno; mentre il tavolo B è ovale $e$ moderno; è fatto di vetro e metallo.

La bici A è una bici da corsa da nomo mentre la bici B è da città da donna; ha anche un cestino; quella $C$ è una bici da bambino con le rotelle. Tutte e tre sono nuove e veloci.

Un prompt poco generativo è, a nostro dire, anche il seguente (p. 107). Se qualcuno ci chiedesse di svolgere lo stesso task in lingua madre non disporremmo di molti argomenti da condividere: 
QUALI MEZZI USI PER COMUNICARE CON LA TUA FAMIGLIA E CON I TUOI AMICI? PREFERISCI USARE L'EMAIL, FACEBOOK, SKYPE O IL TELEFONO? PERCHÉ? SCRIVI AL MASSIMO 50 PAROLE.

Scrive a riguardo McNamara (2000: 53): “[...] If the topic does not match their interests [vale a dire l'interesse degli esaminandi] or is one about which they have little knowledge, the performance is likely to appear less impressive than when candidates are speaking [writing, nel nostro caso] about a more familiar topic [...]". Tornando al nostro esempio, è plausibile il candidato abbiapiù argomenti per descrivere familiari e amici di quanti non ne abbia in merito all'uso di Skype o di Facebook per comunicare con loro (posto che sia iscritto a Facebook e che usi Skype). Anche il prompt del task che segue è ricco di presupposti:

NELLA CLASSE DI TUO FIGLIO CI SONO BAMBINI STRANIERI? ELENCA A QUALI NAZIONALITÀ APPARTENGONO E QUALE RELIGIONE PRATICANO? SCRIVI 25-30 PAROLE.

Si dà, infatti, per scontato cheil candidato abbia figli, che ci siano numerosi compagni stranieri nella classe dell'ipotetico figlio, che si sia a conoscenza delle religioni professate dalle famiglie di costoro. Ravvisiamo, peraltro, due ulteriori criticità nella prova appena descritta:

- il fatto che un elenco di aggettivi (nazionalità, religione) non possa essere considerato un compito di scrittura (cfr. \2.17.,Sottorappresentazione del costrutto);

- la presenza di un refuso (cfr. 2.25.) che può generare confusione: la seconda frase è espressa in forma interrogativa ("Elenca a quali nazionalità appartengono $e$ quale religione praticano?'), laddove dovrebbe essere, invece, espressa in forma affermativa.

Laddove prompt poco generativi siano poco chiari e si accompagnino a immagini di scarsa risoluzione, la situazione si aggrava esponenzialmente, rendendo il compito di difficile risoluzione. Si veda il seguente task. (p. 93). 
GUARDA L'IMMAGINE E DESCRIVI L'ABBIGLIAMENTO

DI ALMENO DUE DEI RAGAZZI RAPPRESENTATI: PORTANO LA GONNA O I PANTALONI? È IN BLANCO O IN NERO... C'È DISEGNATO QUALCOSA SOPRA LE MAGLIETTE CHE INDOSSANO? USANO LA GLACCA? SCRIVI 20-25 PAROLE.

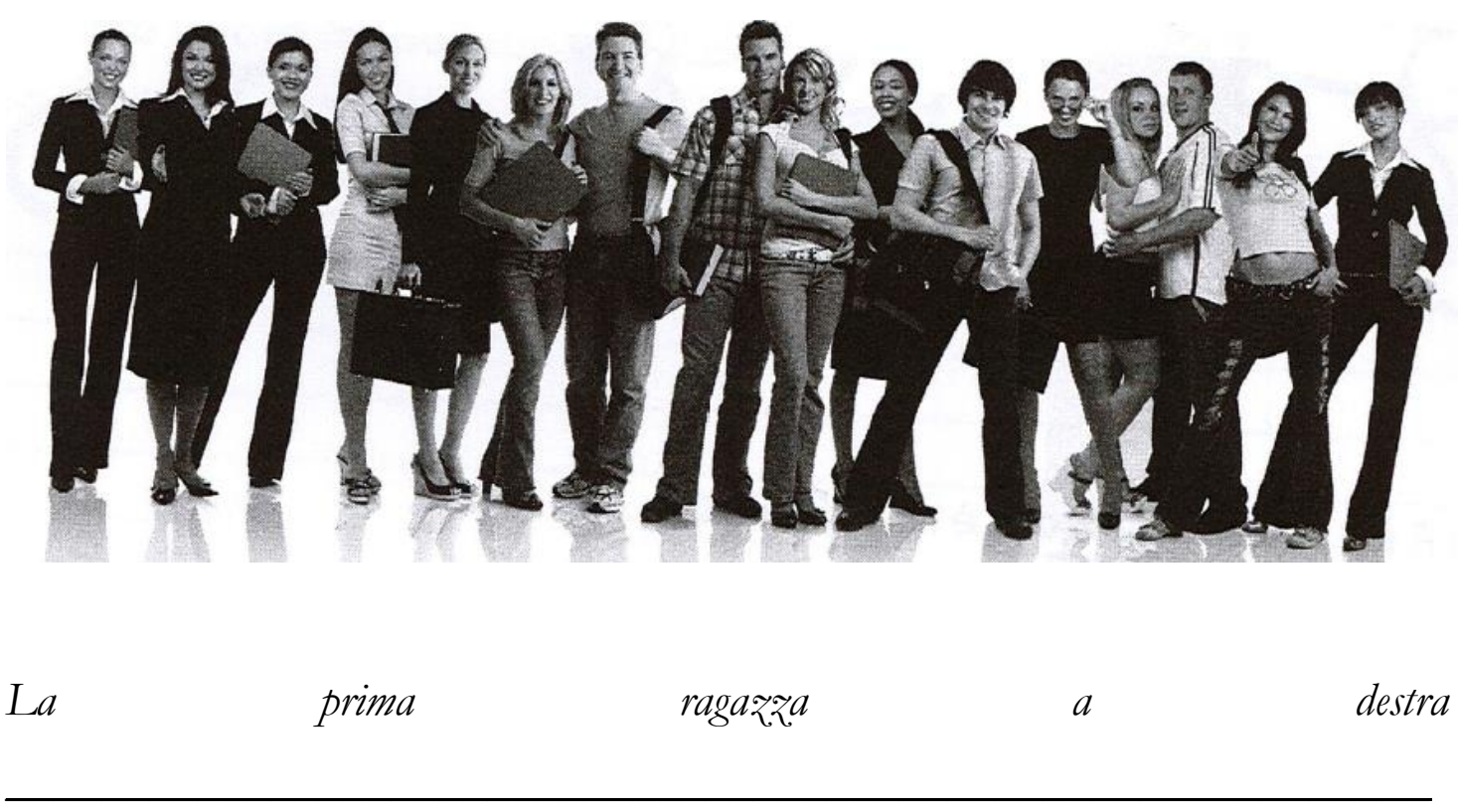

Benché il compito sia linguisticamente facilitato per via del fatto che nella consegna viene fornito il lessico di cui si ha bisogno (pantoloni, gonna, giacca, nero, magliette), l'immagine è confusa: alcuni personaggi sono nascosti da altri, il disegno impresso sulla maglietta della seconda ragazza a destra resiste ad ogni tentativo di decifraggio, ecc. Inoltre, perché così tanti ragazzi quando ne basterebbe un numero minore, ma con una risoluzione migliore delle immagini?

\subsection{Mancata calibrazione}

Il seguente cloze (p. 82-83) è presentato come una prova di comprensione (in realtà, così come è impostato, testa competenze lessicali e morfologiche). 
Focalizziamoci sull'item \#1. La chiave sta nell'avverbio "bene"; i distrattori "ottimo" e "migliore" sono due aggettivi superlativi organici che uno studente di livello A2 non è in grado di gestire; il loro possesso avviene solo al livello B1. Così infatti si evince nel Profilo (Spinelli, Parizzi 2011), vale a dire nello strumento voluto dal Consiglio d'Europa che consente di orientare il valutatore (così come il progettista e l'autore di materiali didattici) nella definizione di quale lessico e quali strutture caratterizzino i primi 4 livelli del quadro (A1, A2, B1, B2).

Lo stesso si può affermare per il distrattore "siano" presente nell'item \#5.

SCEGLI L'ALTERNATIVA CHE COMPLETA CORRETTAMENTE IL BRANO

Caro Andrea, grazie per la tua lunga email. Sto bene, grazie. Adesso lavoro part-time come commessa in un negozio di scarpe, e mi trovo molto (1 ). $M i$ piace stare a contatto con la gente! Poi di sera, due volte alla settimana, vado a nuotare in $(2$ ) con Anna; ti ricordi di lei? Fa l'istruttrice (3 nuoto. Perché non vieni anche tu con (4 )? Se non ricordo male, $(5$ un ottimo

nuotatore.

Ci vediamo presto.

1.

\section{a. migliore}

\section{b. ottimo}

c. bene

2.
a. campo
b. piscina
c. stadio 
3.
a. $d i$
b. con
c. $s u$

4.
a. lui
b. loro
c. noi

5.

a. $s e i$

\section{b. siano}

c. sono

Della mancata calibrazione ci pare ci sia una certa evidenza anche nello svolgimento dei compiti da parte delle autrici, nell'intenzione di offrire un esempio al candidato.

Nella seguente email composta dalle autrici (parte con fondino grigio),sulla base del prompt (parte senza fondino), compaiono tipologie di verbi che nel Profilo figurano al livello B1 (*) e al livello B2 (**)!

\begin{tabular}{|c|c|c|}
\hline A: Antonio:Rossi@libero.it & Re: Week end & 15:05 \\
\hline \multicolumn{3}{|c|}{$\begin{array}{l}\text { Ciao Antonio, } \\
\text { grazie, il fine settimana è stato molto bello. Ora ti racconto: }\end{array}$} \\
\hline \multicolumn{3}{|c|}{$\begin{array}{l}\text { sabato mattina, appena siamo arrivati con il treno, siamo corsi }\left(^{*}\right) \\
\text { subito al mare, dove abbiamo passato tutta la giornata. Domenica, } \\
\text { poi, abbiamo visitato l'acquario di Genova. L'albergo era } \\
\text { accogliente e pulito, anche se Irene e i bambini avrebbero } \\
\text { preferito }\left(^{*}\right) \text { il campeggio. }\end{array}$} \\
\hline \multicolumn{3}{|l|}{ Ci vediamo presto! } \\
\hline & & \\
\hline
\end{tabular}




\subsection{Inattualità dei testi da redigere}

La redazione di una cartolina, per quanto si riferisca a un tipo di testo alla portata di un apprendente di livello A2, ci pare non più attuale: quanti di coloro che viaggiano oggi scrivono ancora cartoline ai loro cari?

\subsection{Refusi}

Ogni testo dato alle stampe richiede una sorveglianza nella scrittura; i correttori di bozze, e più spesso l'autore, sono chiamati a controllare che non ci siano refusi.

La presenza di un refuso ha una notevole gravità nell'ambito del testing, dal momento che si può supporre che un test taker sia indotto a non scegliere una opzione considerata esatta dall'item writer perché non corretta ortograficamente. Si veda il test a scelta multipla che segue (p. 109):

\section{SCEGLI LA RISPOSTA ESATTA. TRA QUALI PERSONE}

$$
\text { PUOI ASCOLTARE QUESTI DIALOGHI? }
$$

2.
a. tra na parrucchiera e un cliente
b. tra un panettiere e un cliente
c. tra n vigile e un autista

\section{CONCLUSIONI}

La vasta presenza di aspetti critici riscontrati nel testo oggetto di indagine ci rende consapevoli dell'estrema cura che la confezione di una prova richiede. Non solo, da più parti si insiste sul processo cooperativo che un'operazione di test designcomporta. Alderson et al. (1995, p.63) sostengono:

"It is [...] absolutely crucial in all test development, for whatever purpose, at whatever level of learner ability and however trivial the consequences of failure on the test might be, that some person or persons other than the individual item writer look closely at each item, respond to the item a student would, reflect upon what abilities are required for successful completion of the 
item/task, and then compare what he or she thinks the item is testing with what the item writer claim it tests".

Da parte nostra siamo dell'opinione che, per quanto la stesura de "Il test di italiano per stranieri" appaia frutto di un lavoro a sei mani, una collaborazione più stretta tra gli autori (ed eventualmente con esperti esterni) avrebbe portato a prove più accurate. Questo è del resto il nostro auspicio rivolto a tutti coloro che si accingono a preparare dei test. un'accuratissima revisione tra pari è il primo passo verso una prova solida ed efficace.

\section{Riferimenti Bibliografici}

ALDERSON, J. C.; CLAPHAM, C.; WALL, D. Language Test Construction and Evaluation. Cambridge: Cambridge University Press, 1995.

AVELlA, P. BORGONOVO, P. DESIDERIO F. Il test di italiano per stranieri. Milano: Alpha Test, 2013.

CANGELOSI, J. S.Designing Tests for Evaluating Student Acbievement. New York: Longman, 1990.

CARR, N. T. Designing and Analyzing Language Tests. Oxford: Oxford University Press, 2011.

COUNCILOFEUROPE. Common European Framework of Reference for Languages: Learning, Teaching, Assessment, <http://www.coe.int/t/dg4/linguistic/Source/Framework_EN.pdf>, 2001.

HALADYNA, T. M.; DOWNING, S. M., RODRIGUEZ, M. C. "A Review of Multiple-choice Item-writing Guidelines for Classroom Assessment", Applied Measurement in Education, 2002. 15, 3, 309-334. 
HEATON, J. B. Writing English Language Tests. Longman: Londra, 1988.

KEENAN, J. M.; BETJEMAN, R. S.; OLSON, R. K. "Reading Comprehension Tests Vary in the Skills They Assess: Differential Depending on Decoding and Oral Comprehension", Scientific Studies of Reading, 2008. 12, 3, p. 281-300.

McNAMARA, T. Language Testing, Oxford: Oxford University Press, 2000.

SPINELLI, B.; PARIZZI, F. Profilo della lingua italiana, Firenza: La Nuova Italia, 2011.

Into Europe Project, British Council, Hungary, 2002. Disponível em: $<$ http://www.lancs.ac.uk/fass/projects/examreform/Media/GL_Reding\&Use.pdf $>$ 